\title{
CARACTERIZACIÓN DE ÓBITO FETAL TARDÍO EN LA CLÍNICA MATERNIDAD RAFAEL CALVO DE CARTAGENA DE INDIAS ENTRE LOS AÑOS 2012 A 2013
}

\author{
CHARACTERIZATION OF LATE STILLBIRTH IN CLÍNICA DE \\ MATERNIDAD RAFAEL CALVO FROM CARTAGENA BETWEEN \\ 2012 AND 2013
}

Monsalve-Montoya Rosa Edith ${ }^{1}$

Méndez-Rodríguez Rogelio ${ }^{2}$

Salcedo-Ramos Francisco ${ }^{2}$

Correspondencia: remm0501@hotmail.com

Recibido para evaluación: abril - 15 - 2015. Aceptado para publicación: octubre - 25-2015.

\section{RESUMEN}

Introducción: el óbito fetal tardío tiene connotaciones de salud pública y es indispensable su estudio, aun cuando no hace parte de los objetivos del milenio. El amplio subregistro, la gran variedad de clasificaciones, la dificultad en la unificación de conceptos han hecho que su búsqueda sea inespecífica. Los estudios sobre sus causas arrojan datos variables.

Objetivo: describir las causas de óbito fetal tardío en las pacientes que consultaron a una clínica obstétrica

Materiales y métodos: estudio descriptivo transversal. La población de estudio fueron 108 casos de pacientes que tuvieron parto en la Clínica Maternidad Rafael Calvo (CMRC), Cartagena de Indias-Colombia, entre los años 2012 a 2013 con diagnóstico de óbito fetal tardío. Se analizaron las siguientes variables: características sociodemográficas (edad, estado civil, zona, escolaridad, ocupación, raza), antecedente obstétrico y clínico de la madre (edad de la paciente, paridad, número de abortos, número de óbitos, número de prematuros, período intergenésico, patologías maternas) y variables obstétricas de su embarazo actual (edad gestacional al momento del diagnóstico de óbito fetal tardío, índice de masa corporal (IMC), número de controles prenatales, momento de ocurrencia, alteraciones del líquido amniótico, alteraciones de la placenta, anomalía del cordón, hemoglobina materna, infecciones, ruptura prematura de membranas, percentil del índice ponderal del feto, características del líquido amniótico). En aquellas con resultados de necropsia se analizó la causa de muerte dada por el laboratorio de patología. Los datos se recolectaron en un formato con las variables estudiadas y el análisis de las variables cualitativas y cuantitativas se realizó con el programa estadístico IBM SPSS (SPSS Inc. Released 2009. PASW Statistics for Windows, Version 18.0. Chicago: SPSS Inc.).

Resultados: se encontró con mayor frecuencia edad materna de 15-20 años (34.3\%), estado civil: unión libre $(86.1 \%)$, zona de residencia urbana $(58.3 \%)$, escolaridad secundaria completa $(42.6 \%)$, ocupación ama de casa $(89.8 \%)$ y raza mestiza $(75.9 \%)$.

Médico. Estudiante de Postgrado. Ginecología y Obstetricia. Facultad de Medicina. Universidad de Cartagena. Colombia.

2 Médico. Especialista en Ginecología y Obstetricia. Docente Departamento de Ginecología y Obstetricia. Facultad de Medicina. Universidad de Cartagena. Colombia. 
ISSN: 2215-7840, 7(1), enero-junio 2016, Monsalve-Montoya Rosa Edith, Méndez-Rodríguez Rogelio,

El $43.5 \%$ de las pacientes reportaron entre 2-3 embarazos previos, sin antecedente de óbito $(55.6 \%)$, ni restricción de crecimiento intrauterino previo (RCIU) $(58.3 \%)$, ni partos pretérmino $(50.9 \%)$. Período intergenésico entre $12-24$ meses $(39.8 \%)$, número de controles prenatales entre 1-4 (44.4\%). El 67.6\% de las pacientes tenían entre 2836.6 semanas de gestación; en el $95.4 \%$ la ocurrencia de la muerte fue en el anteparto, sexo del feto masculino (52.8\%); el peso materno fue normal con un $33.3 \%$, solo el $12 \%$ de las paciente eran obesas. El índice ponderal de los fetos fue del $50 \%$ en el percentil $<10$ y la hemoglobina materna $50.9 \%$ sin anemia. De patologías maternas las infecciones genitourinarias $(20.3 \%)$ y los trastornos hipertensivos $(16.2 \%)$ fueron las más frecuentes. De las pacientes que reportaron patologías, $14(29.7 \%)$ presentaban 2 o más. Se encontró con mayor frecuencia la causa materna $51.4 \%$ : placentaria $35.2 \%$, inexplicada $7.5 \%$ y fetales $5.7 \%$.

Conclusión: las causas de óbito fetal tardío de las pacientes analizadas en CMRC de Cartagena de Indias - Colombia en orden de frecuencia son: causa materna, placentaria, inexplicada y fetal. Rev.cienc.biomed. 2016;7(1):68-79.

\section{PALABRAS CLAVE}

Óbito fetal tardío; Mortinato; Factores de riesgo; Etiología.

\section{SUMMARY}

Introduction: late stillbirth is a public health problem and it is necessary its study, even when it is not included in the millennium development goals. The wide underreporting, variety of classifications, difficulty in the unification of the concepts have made the late stillbirth search something unspecific. The studies about its causes generate variable data.

Objective: to describe the late stillbirth causes in the female patients that consulted an obstetric clinic.

Methods: this is a descriptive-transversal study. The study population was 108 cases of female patients that gave birth in Clínica Maternidad Rafael Calvo (CMRC) in Cartagena de Indias-Colombia between 2012 and 2013 with late stillbirth diagnosis. The following variables were analyzed: sociodemographic characteristics (age, marital status, zone, education, occupation, and race), obstetrical-clinical history of the mother (age of the patient, parity, number of miscarriages, number of deaths, number of premature babies, intergenesic periods and mother's pathologies) and obstetrical variables of the current pregnancy (gestational age at the moment of late stillbirth diagnosis, bodymass index (BMI), number of attendances to prenatal care, moment in which it happened, alterations in amniotic fluid, alterations in the placenta, umbilical cord abnormalities, maternal hemoglobin, infections, premature rupture of membranes, percentile rank of the fetus and amniotic fluid characteristics). The causes of death were analyzed in those women who had necropsy results given by pathological laboratory. Data were collected by means of a survey with the studied variables; the analysis of quantitative and qualitative variables was carried out with the statistical program IBM SPSS (SPSS Inc. Released 2009. PASW Statistics for Windows, Version 18.0. Chicago: SPSS Inc.).

Results: the most frequent maternal age was 15-20 (34.3\%), marital status: cohabiting $(86.1 \%)$, residential area $(58.3 \%)$, complete high school $(42.6 \%)$, housewife $(89.8 \%)$ and mixed race $(75.9 \%)$. The $43.5 \%$ of the female patients affirmed have had between two and three previous pregnancies without clinical record of deaths $(55.6 \%)$ or previous intrauterine growth restriction (58.3\%), no preterm birth $(50.9 \%)$. Intergenesic period between $12-24$ months (39.8\%), number of attendances to prenatal care between one and four $(44.4 \%)$. The $67.6 \%$ of the female patients had between 28 and 36 weeks of gestation; the death was in antepartum period in the $95.4 \%$, male fetus $(52.8 \%)$; maternal weight was normal with $33.3 \%$, only $12 \%$ of the female patients were obese. The ponderal index of the fetuses was $50 \%$ in the percentile $<10$ and maternal hemoglobin $50.9 \%$ without anemia. The most frequent maternal pathologies were genitourinary infections (20.3\%) and hypertensive disorders (16.2\%). Among the female patients that presented pathologies 14 (29.7\%) of them presented two or more. The most frequent causes were maternal (51.4\%): placental $35.2 \%$, unexplained $7.5 \%$ and fetal $5.7 \%$.

Conclusion: the most frequent causes of late stillbirth in the female patients analyzed at CMRC in Cartagena-Colombia were maternal cause, placental, unexplained and fetal. Rev.cienc.biomed. 2016;7(1):68-79.

\section{KEYWORDS}

Late fetal death; Late stillbirth; Risk factors; Etiology. 


\section{INTRODUCCIÓN}

La Organización Mundial de la Salud (OMS) define óbito fetal como "la muerte antes de la expulsión o extracción completa de la madre, del producto de la concepción, cualquiera haya sido la duración de la gestación" y a su vez, direcciona a cada país a establecer la edad gestacional del óbito para fines de notificación (1). Además, define de acuerdo a la edad gestacional en el momento del parto el óbito temprano cuando ocurre entre las 22-27 semanas de edad gestacional, y óbito tardío cuando ocurre de la semana 28 de edad gestacional en adelante. Aunque la división es algo arbitraria, esta clasificación permite unificar conceptos y dividir los óbitos difíciles de prevenir (temprano) de los potencialmente evitables (tardíos). En Colombia, el Departamento Administrativo Nacional de Estadística (DANE) acoge la definición dada por la OMS (2).

A pesar de la atención creciente e inversión para problemas maternos, neonatales y de la infancia, el óbito fetal continúa siendo "invisible": no está dentro de las metas del milenio, no es investigado por la OMS, ni cuantificado en la epidemiología mundial. La reducción anual de óbito fetal ha sido más lenta que la reducción de mortalidad materna e infantil: $1.1 \%$ entre 1995 y 2009 . Si no se toman medidas a nivel mundial, se estima que para el año 2020 , más del $90 \%$ de todos los óbitos se presentarán en Asia del Sur y África Subsahariana $(1,3)$.

La incidencia de óbito fetal en Estados Unidos en el año 2005 fue de 6.2/1.000 nacimientos (4). Las tasas de óbito fetal en los países subdesarrollados son más altas que en los países desarrollados: 9-34/1.000 nacimientos vs. 3/1.000 nacimientos $(3,5)$.

En todo el mundo, hay entre tres y cuatro millones de mortinatos cada año, el $98 \%$ de estas muertes se producen en el mundo en desarrollo (6). A nivel de América Latina y el Caribe el área geográfica que presenta una mayor tasa de mortalidad perinatal es el Caribe latino (6).

A nivel nacional, hay un amplio subregistro de mortalidad fetal por la poca notificación del evento. Según la Encuesta Nacional de Demografía y Salud (EDNS-2005) la tasa de mortalidad perinatal fue de 17 muertes por mil embarazos de siete o más meses de gestación, con un número de muertes neonatales tempranas mayor que el número de mortinatos (6). En el año 2013 se reportaron 44.620 muertes fetales, de las cuales 1.334 se notificaron en el departamento de Bolívar (7). En la Clínica de Maternidad Rafael Calvo (CMRC) de la ciudad de Cartagena de Indias-Colombia, durante el año 2011 (datos no publicados), hubo 8.427 partos (vaginales y por cesárea) y de estos, 123 casos de muertes fetales fueron notificados.

Aunque se conocen factores de riesgo para óbito fetal que pueden detectarse desde el control prenatal, muchas pacientes no los reportan, y por esta razón, no se han podido desarrollar modelos clínicamente útiles para la estimación específica de riesgos para óbito fetal por cada paciente. Dentro de los factores de riesgo reportados en los estudios se encuentran: raza negra, zona rural de residencia, violencia intrafamiliar, trauma, tabaquismo, nivel educativo y socioeconómico bajo, edad materna mayor de 35 años, obesidad, enfermedades maternas, multiparidad, pobre control prenatal, período intergenésico corto, embarazo múltiple, embarazo prolongado, antecedentes de óbito, parto pretérmino y restricción en el crecimiento intrauterino (RCIU) (8-11).

Las causas de óbito fetal se sobreponen a los factores de riesgo, pero pueden agruparse en fetales, placentarias, maternas e inexplicadas. Clasificar las causas de óbito fetal en un solo ítem es difícil, pues muchas muertes fetales pueden ser multicausales, por ejemplo, anomalías placentarias como el desprendimiento de placenta puede estar asociado con hipertensión arterial, o la insuficiencia placentaria con alguna aneuploidía (12).

En los países desarrollados las causas son diferentes a las de países subdesarrollados $(13,14)$. En estos últimos las causas más frecuentes de óbito fetal son el parto obstruido/prolongado, preeclampsia y las infecciones, mientras que en países desarrollados son alteraciones del cariotipo, malformaciones congénitas, patologías de la 
ISSN: 2215-7840, 7(1), enero-junio 2016, Monsalve-Montoya Rosa Edith, Méndez-Rodríguez Rogelio,

Salcedo-Ramos Francisco

placenta que llevan a RCIU y las patologías maternas $(15,16)$.

La causa de óbito también depende de la edad gestacional. En una revisión se encontraron que las causas de óbito fetal temprano eran la infección (19\%), desprendimiento prematuro de placenta normoinserta (14\%) y anomalías fetales (14\%) (17). En óbito fetal tardío se ha reportado la causa inexplicable como la más frecuente (18-20). No identificar una causa específica se puede atribuir a varios factores: en primer lugar, el feto en muy pocos casos es sometido a estudio anatomopatológico completo, ya sea por falta de conocimiento médico, de disponibilidad de pruebas, o por temor o creencias culturales y religiosas de la familia; segundo, la falta de mecanismos en las instituciones para estudiar a los fetos. El objetivo de este estudio es describir las causas de óbito fetal tardío en las pacientes que consultaron a una clínica obstétrica.

\section{MATERIALES Y MÉTODOS}

Estudio descriptivo prospectivo transversal. La población de estudio fueron 108 casos de pacientes embarazadas que tuvieron parto vaginal o por cesárea en la CMRC en Cartagena de Indias-Colombia entre los años 20122013, a quienes se les diagnosticó óbito fetal tardío, independientemente del momento y lugar de ocurrencia de la muerte.

En todas las pacientes se analizaron las siguientes variables: características sociodemográficas (edad, estado civil, zona, escolaridad, ocupación, raza), antecedentes obstétricos y clínicos de la madre (edad de la paciente, paridad, número de abortos, número de partos (vaginal o cesárea), número de hijos vivos, número de óbitos, número de prematuros, período intergenésico, patologías maternas, edad gestacional al momento del diagnóstico del óbito, índice de masa corporal (IMC), número de controles prenatales, momento de ocurrencia (anteparto o intraparto), alteraciones de la placenta, anomalía del cordón, hemoglobina materna en el último trimestre, infecciones, ruptura prematura de membranas, percentil del índice ponderal del nacido. En aquellas con resultados de necropsia se analizó la causa de muerte dada por el laboratorio de patología.

En el presente estudio se clasificó la hemoglobina materna $(\mathrm{mg} / \mathrm{dl})$ según las directrices de la OMS (21) donde > o igual a 11 es sin anemia, de 10 a 10.9 es anemia leve, de 7 a 9.9 es moderada y menor de 7 es anemia grave.

Un indicador útil para evaluar el estado de las gestantes es el índice de masa corporal (IMC) pues determina qué tan adecuado se encuentra el peso de la paciente con relación a su estatura $(22,23)$. El IMC se obtiene a partir de dividir el peso del individuo en kilogramos $(\mathrm{Kg})$ sobre la estatura en metros al cuadrado, así: IMC = Peso (Kg)/Estatura $\left(\mathrm{m}^{2}\right)$. En el estudio se utilizó el IMC según la semana de gestación, propuesta por Atalah et al., para clasificar el estado nutricional de las pacientes donde enflaquecida es $<20.0$, normal de 20.0-24.9, sobrepeso de 25-29.9 y obesidad $>30.0$ (24).

Se utilizó el índice ponderal (IP) pues ayuda a identificar al recién nacido con poca cantidad de tejido graso y de masa muscular, aun si el peso al nacer es adecuado para la edad gestacional (25). El IP se calcula con la siguiente fórmula: IP = peso al nacer (gramos)/talla $(\mathrm{cm})^{3} \times 100$. El resultado se lleva a una curva para ambos sexos ya que el género no afecta el IP; los investigadores del CLAP-/OPS/OMS (Centro Latinoamericano de Perinatología de la OPS/OMS) establecieron los percentiles 10, 50 y 90 del IP según edad gestacional entre las 33 y 42 semanas. Se considera que los recién nacidos con IP por debajo del percentil 10 para la edad gestacional presentan malnutrición in útero (26).

La insuficiencia placentaria es un concepto obstétrico que incluye embarazo sin patología materno-fetal evidente, con oligoamnios, restricción del crecimiento fetal y asociado con infartos $y / 0$ desprendimiento de placenta (27-28).

La participación de las pacientes fue de forma voluntaria para brindar la información solicitada, por lo tanto, previa explicación del estudio se realizó firma de consentimiento informado. Los datos se recolectaron en 
un formato con las variables estudiadas. La fuente primaria de la información fueron la paciente y sus acompañantes por medio de interrogatorio; también por medio de la historia clínica para datos de laboratorio y ecografías, y el resultado de necropsia entregado por el área de patología de la misma institución en los casos en que se practicó estudio al feto y/o placenta. El análisis de las variables cualitativas y cuantitativas se realizó con el programa estadístico IBM SPSS (SPSS Inc. Released 2009. PASW Statistics for Windows, Version 18.0. Chicago: SPSS Inc.).

\section{RESULTADOS}

En la CMRC en el año 2012 se atendieron 9.400 partos (4.880 vaginales y 4.520 por cesárea), y se reportaron 120 muertes fetales. En el año 2013, se atendieron 9.011 partos (4.713 vaginales y 4.298 por cesárea) y se reportaron 103 muertes fetales (datos no publicados). En total, entre los años 2012 y 2013 se reportaron 18.411 partos y 223 óbitos fetales, que corresponden al 1.2\% de los partos atendidos. Durante el período de estudio se lograron incluir 108 casos de óbito fetal tardío que corresponden al $48.4 \%$ de los óbitos fetales reportados. Estos 108 casos se distribuyeron así: 47 en el 2012 y 61 en el 2013, en ese período se realizó el análisis.

El grupo etario de mayor frecuencia fue de $15-20$ años (34.3\%). En cuanto al estado civil, unión libre $(86.1 \%)$, la zona de residencia urbana $(58.3 \%)$, la escolaridad secundaria completa (42.6\%) y técnico $(9.3 \%)$ suman un total de $51.9 \%$, la ocupación ama de casa $(89.8 \%)$ y la raza mestiza $(75.9 \%)$ fueron los resultados más frecuentes. Antecedentes tóxicos (fumar, licor o sustancias psicoactivas) y de maltrato físico intrafamiliar o trauma fueron negados por todas las pacientes (Tabla No 1 ).

Entre los antecedentes obstétricos y clínicos de las pacientes incluidas en este estudio se reportó con más frecuencia que el $43.5 \%$ de las pacientes tuvieron 2-3 embarazos previos. De las pacientes con embarazos anteriores se encontró que no reportaban óbito (55.6\%), ni RCIU (58.3\%), ni partos pretérmino previos $(50.9 \%)$. El período inter-

\begin{tabular}{|c|c|c|c|}
\hline \multicolumn{4}{|c|}{$\begin{array}{c}\text { TABLA No } 1 . \\
\text { CARACTERÍSTICAS SOCIODEMOGRÁFICAS } \\
n=108\end{array}$} \\
\hline \multicolumn{2}{|c|}{ Variable } & 108 & $\%$ \\
\hline \multirow{6}{*}{ Edad (años) } & $<15$ & 2 & 1.9 \\
\hline & $15-20$ & 37 & 34.3 \\
\hline & $21-25$ & 35 & 32.4 \\
\hline & $26-30$ & 22 & 20.4 \\
\hline & $31-35$ & 7 & 6.5 \\
\hline & $>35$ & 5 & 4.6 \\
\hline \multirow{5}{*}{ Estado civil } & Unión libre & 93 & 86.1 \\
\hline & Soltera & 11 & 10.2 \\
\hline & Casada & 4 & 3.7 \\
\hline & Viuda & 0 & 0 \\
\hline & Separada & 0 & 0 \\
\hline \multirow{2}{*}{ Zona } & Rural & 45 & 41.7 \\
\hline & Urbana & 63 & 58.3 \\
\hline \multirow{6}{*}{ Escolaridad } & Ninguna & 2 & 1.9 \\
\hline & $\begin{array}{l}\text { Primaria } \\
\text { incompleta }\end{array}$ & 12 & 11.1 \\
\hline & $\begin{array}{l}\text { Primaria } \\
\text { completa }\end{array}$ & 10 & 9.3 \\
\hline & $\begin{array}{l}\text { Secundaria } \\
\text { incompleta }\end{array}$ & 28 & 25.9 \\
\hline & $\begin{array}{c}\text { Secundaria } \\
\text { completa }\end{array}$ & 46 & 42.6 \\
\hline & Técnico & 10 & 9.3 \\
\hline \multirow{4}{*}{ Ocupación } & Ama de casa & 97 & 89.8 \\
\hline & $\begin{array}{c}\text { Empleada } \\
\text { independiente }\end{array}$ & 4 & 3.8 \\
\hline & Oficios varios & 2 & 1.9 \\
\hline & Estudiante & 5 & 4.6 \\
\hline \multirow{2}{*}{$\begin{array}{l}\text { Antecedentes } \\
\text { tóxicos }\end{array}$} & Sí & 0 & 0 \\
\hline & No & 108 & 100 \\
\hline \multirow{2}{*}{$\begin{array}{l}\text { Antecedentes } \\
\text { de maltrato }\end{array}$} & Sí & 0 & 0 \\
\hline & No & 108 & 100 \\
\hline \multirow{4}{*}{ Raza } & Mestiza & 82 & 75.9 \\
\hline & Negra & 25 & 23.1 \\
\hline & Blanca & 1 & 0.9 \\
\hline & Indígena & 0 & 0 \\
\hline
\end{tabular}




\begin{tabular}{|c|c|c|c|c|c|c|c|}
\hline \multicolumn{8}{|c|}{$\begin{array}{c}\text { TABLA No } 2 . \\
\text { CARACTERÍSTICAS OBSTÉTRICAS } \\
\text { N=108 }\end{array}$} \\
\hline \multicolumn{2}{|c|}{ Variable } & \multirow{2}{*}{$\frac{N}{40}$} & \multirow{2}{*}{$\begin{array}{l}\% \\
37\end{array}$} & \multicolumn{2}{|c|}{ Variable } & \multirow{2}{*}{$\frac{N}{103}$} & \multirow{2}{*}{$\begin{array}{c}\% \\
95.4\end{array}$} \\
\hline \multirow{4}{*}{ Paridad } & Primigestante & & & \multirow{2}{*}{$\begin{array}{l}\text { Momento de } \\
\text { ocurrencia }\end{array}$} & Anteparto & & \\
\hline & $1-3$ & 47 & 43.5 & & Intraparto & 5 & 4.6 \\
\hline & $4-5$ & 11 & 10.2 & \multirow{3}{*}{ Sexo del feto } & Masculino & 57 & 52.8 \\
\hline & 6 o más & 10 & 9.3 & & Femenino & 49 & 45.4 \\
\hline \multirow{5}{*}{$\begin{array}{l}\text { Período } \\
\text { intergenésico } \\
\text { (en meses) }\end{array}$} & $<=11$ & 10 & 9.3 & & No determinado & 2 & 1.9 \\
\hline & $12-24$ & 43 & 39.8 & \multirow{4}{*}{$\begin{array}{l}\text { Características } \\
\text { líquido amniótico }\end{array}$} & Hemático & 23 & 21.3 \\
\hline & $25-36$ & 7 & 6.5 & & Meconio & 29 & 26.9 \\
\hline & 37 o más & 7 & 6.5 & & Claro & 53 & 49.1 \\
\hline & No aplica & 41 & 38 & & No registra & 3 & 2.8 \\
\hline \multirow{3}{*}{ Óbito previo } & Sí & 8 & 7.4 & \multirow{4}{*}{$\begin{array}{l}\text { Clasificación } \\
\text { del estado } \\
\text { nutricional } \\
\text { materno }\end{array}$} & Enflaquecida & 33 & 30.6 \\
\hline & No & 60 & 55.6 & & Normal & 36 & 33.3 \\
\hline & No aplica & 40 & 37 & & Sobrepeso & 26 & 24.1 \\
\hline \multirow{3}{*}{ RCIU previo } & Sí & 5 & 4.6 & & Obesa & 13 & 12 \\
\hline & No & 63 & 58.3 & \multirow{5}{*}{$\begin{array}{l}\text { Hemoglobina } \\
\text { materna } \\
(\mathrm{mg} / \mathrm{dl})\end{array}$} & Sin anemia & 55 & 50.9 \\
\hline & No aplica & 40 & 37 & & Anemia leve & 23 & 21.3 \\
\hline \multirow{3}{*}{$\begin{array}{l}\text { Parto pretérmino } \\
\text { previo }\end{array}$} & Sí & 13 & 12 & & Anemia & 29 & 269 \\
\hline & No & 55 & 50.9 & & moderada & & \\
\hline & No aplica & 40 & 37 & & Anemia severa & 1 & 0.9 \\
\hline \multirow{3}{*}{$\begin{array}{l}\text { Número de } \\
\text { controles } \\
\text { prenatales }\end{array}$} & 0 & 21 & 19.4 & \multirow{6}{*}{$\begin{array}{l}\text { Percentil de } \\
\text { índice ponderal } \\
\text { fetal }\end{array}$} & $<10$ & 54 & 50 \\
\hline & $1-4$ & 48 & 44.4 & & 10 & 5 & 4.6 \\
\hline & $>5$ & 39 & 36.1 & & 50 & 23 & 21.3 \\
\hline \multirow{3}{*}{$\begin{array}{l}\text { Edad gestacional } \\
\text { (semanas) }\end{array}$} & $28-36.6$ & 73 & 67.6 & & 90 & 14 & 13 \\
\hline & $37-41.6$ & 33 & 30.6 & & $>90$ & 11 & 10.2 \\
\hline & 42 o más & 2 & 1.9 & & No determinado & 1 & 0.9 \\
\hline
\end{tabular}

genésico entre $12-24$ meses (39.8\%), y el número de controles prenatales entre 1-4 (44.4\%) (Tabla No 2).

Con respecto al embarazo actual al momento del diagnóstico de óbito fetal tardío se encontró: $67.6 \%$ de los casos entre 28- 36.6 semanas de gestación, $95.4 \%$ momento de ocurrencia de la muerte anteparto, sexo del feto masculino $(52.8 \%)$. Con respecto a la clasificación del estado nutricional materno, para las pacientes del estudio se encontró en el $33.3 \%$ clasificación normal, $24.1 \%$ con sobrepeso, solo $12 \%$ eran obesas. Según el percentil de IP de los fetos se observó el $50 \%$ en el percentil $<10$.

Se encontró que el $50.9 \%$ de las maternas estaban sin anemia; los tres tipos de anemia (leve, moderada y severa) en conjunto suman $49.1 \%$ (Tabla No 2). Se encontraron dos casos con hemoglobina mayor de 14 y un caso con hemoglobina menor de siete, luego, al analizar el peso fetal de estas tres pacientes se encontró índice ponderal por debajo del percentil 10 . 
De las patologías maternas presentes al momento del óbito fetal tardío se encontró con más frecuencia: $49.6 \%$ sin ninguna patología, $20.3 \%$ infecciones genitourinarias, $16.25 \%$ trastornos hipertensivos. Es importante anotar que se encontró que de las 47 pacientes con patologías, 14 de ellas (29.78\%) presentaban dos o más patologías.

De los embarazos gemelares $(\mathrm{N}=4)$ se encontró que todas las pacientes eran primigestantes con promedio de edad gestacional de 35 semanas sin ninguna patología previa conocida y con promedio de controles prenatales de cuatro, tres de ellos con índice ponderal por debajo del percentil 10; también se encontró que el $75 \%$ de los casos eran monocoriales biamnióticos. Solo a uno le hicieron necropsia que concluyó con hipoperfusión placentaria no especificada (Tabla No 3 ).

\begin{tabular}{|c|c|c|c|}
\hline \multicolumn{4}{|c|}{$\begin{array}{c}\text { TABLA No } 3 . \\
\text { PATOLOGÍAS MATERNAS } \\
\text { N }=108\end{array}$} \\
\hline & & $\mathrm{N}$ & $\%$ \\
\hline \multirow{2}{*}{$\begin{array}{l}\text { Trastornos } \\
\text { hipertensivos }\end{array}$} & HTA crónica & 6 & 4.8 \\
\hline & Preeclampsia & 14 & 11.3 \\
\hline \multirow{2}{*}{ Diabetes } & Gestacional & 3 & 2.4 \\
\hline & Pregestacional & 2 & 1.6 \\
\hline \multirow{3}{*}{$\begin{array}{l}\text { Infecciones } \\
\text { genitourinarias }\end{array}$} & $\begin{array}{l}\text { Infección } \\
\text { urinaria }\end{array}$ & 15 & 12.2 \\
\hline & Sífilis & 4 & 3.2 \\
\hline & Otras * & 6 & 4.8 \\
\hline \multicolumn{2}{|l|}{ Epilepsia } & 2 & 1.6 \\
\hline \multicolumn{2}{|l|}{ Asma } & 1 & 0.8 \\
\hline \multirow{2}{*}{$\begin{array}{l}\text { Embarazo } \\
\text { múltiple } \\
\text { (gemelar) }\end{array}$} & $\begin{array}{l}\text { Monocorial } \\
\text { biamniótico }\end{array}$ & 3 & 2.4 \\
\hline & $\begin{array}{l}\text { Bicorial } \\
\text { biamniótico }\end{array}$ & 1 & 0.8 \\
\hline \multicolumn{2}{|l|}{ Hipertiroidismo } & 1 & 0.8 \\
\hline \multirow{2}{*}{ Anemia } & Nutricional & 3 & 2.4 \\
\hline & Falciforme & 1 & 0.8 \\
\hline \multicolumn{2}{|l|}{ Ninguna } & 61 & 49.6 \\
\hline
\end{tabular}

*Se encontró condiloma vulvar, vaginosis y sepsis de origen placentario.
Como causas maternas, según la literatura, se incluyen múltiples variables (edad > 35 años, obesidad, patologías maternas, drogas, alcohol, tabaquismo, infecciones, parto pretérmino actual, trabajo de parto distócico, ruptura uterina, embarazo prolongado) (12); en este estudio para óbito fetal tardío se encontró que 89 pacientes $(82.4 \%)$ tenían una o varias. Sumado a eso, en el grupo de pacientes de causa materna se encontró con mayor frecuencia el parto pretérmino $73(50.6 \%)$ y las patologías maternas 51 (35.4\%) (Tabla No 4).

\begin{tabular}{|l|c|c|}
\hline \multicolumn{3}{|c|}{ TABLA No 4. } \\
CAUSAS MATERNAS DE ÓBITO FETAL TARDío \\
\hline \multicolumn{3}{|c|}{$\mathbf{1 0 8}$} \\
\hline Edad mayor 35 años & N & $\%$ \\
\hline Obesidad & 5 & 3.4 \\
\hline Una o más patologías maternas & 13 & 9.0 \\
\hline Parto pretérmino (28-36.6 semanas) & 51 & 35.4 \\
\hline Embarazo prolongado & 2 & 50.6 \\
\hline Drogas, alcohol, tabaquismo & 0 & 0 \\
\hline Ninguna & 19 & 13.2 \\
\hline
\end{tabular}

Como causas placentarias de óbito tardío se encontraron con más frecuencia: $38.7 \%$ ninguna, $22.5 \%$ insuficiencia placentaria, $17.7 \%$ DPPNI, $8.1 \%$ accidentes del cordón y $8.1 \%$ corioamnionitis y $4.8 \%$ ruptura prematura de membranas (Tabla No 5 ).

\begin{tabular}{|c|c|c|c|}
\hline \multicolumn{4}{|c|}{$\begin{array}{l}\text { TABLA No } 5 \text {. } \\
\text { CAUSAS PLACENTARIAS DE ÓBITO FETAL TARDÍO } \\
\text { N=108 }\end{array}$} \\
\hline & & $\mathrm{N}$ & $\%$ \\
\hline \multicolumn{2}{|c|}{ Ruptura prematura de membranas } & 6 & 4.8 \\
\hline \multicolumn{2}{|c|}{ Desprendimiento prematuro de placenta } & 22 & 17.7 \\
\hline \multicolumn{2}{|c|}{ Placenta previa } & 0 & 0.0 \\
\hline \multicolumn{2}{|c|}{ Corioamnionitis } & 10 & 8.1 \\
\hline \multicolumn{2}{|c|}{ Insuficiencia placentaria } & 28 & 22.5 \\
\hline \multicolumn{2}{|c|}{ Hemorragia feto-materna } & 0 & 0.0 \\
\hline \multirow{4}{*}{$\begin{array}{l}\text { Accidentes } \\
\text { del cordón }\end{array}$} & Prolapso & 2 & 1.6 \\
\hline & Nudo del cordón & 1 & 0.8 \\
\hline & Doble circular & 4 & 3.2 \\
\hline & Inserción marginal & 2 & 1.6 \\
\hline \multicolumn{2}{|c|}{ Asfixia intraparto } & 1 & 0.8 \\
\hline \multicolumn{2}{|l|}{ Ninguna } & 48 & 38.7 \\
\hline
\end{tabular}


Como causas fetales de óbito fetal tardío se encontró en el estudio que el $89.1 \%$ no reportaron ninguna causa fetal, el $7.3 \%$ reportaron malformaciones, el $3.6 \%$ hidropesía no inmune y ninguno con infecciones.

Con causa inexplicable se encontraron 13 pacientes. Al revisar estas pacientes con causa inexplicada de óbito se encontró que solo una de ellas tenía necropsia y que no reportó causa de muerte, pues no recibió disco placentario, $y$, en 10 de ellas, fetos con índice ponderal debajo del percentil 10, lo cual puede mostrar la importancia de haberse realizado necropsia puesto que la malnutrición intraútero de estos fetos podría haberlas clasificado de una manera diferente reduciendo el porcentaje de causa inexplicable. Además, al revisar las pacientes con causa inexplicable se encontró que todas reportaban factores de riesgo (sobrepeso 3, raza negra 4, residencia rural 9, escolaridad incompleta 7, primigestantes 7, mal control prenatal 7, período intergenésico corto 3, anemia 7 , y 1 paciente con hemoglobina materna de 14).

En resumen todas las causas de óbito fetal tardío para este estudio (recordando que muchas pacientes reportan multicausalidad), se dieron en orden de frecuencia así: causa materna (51.4\%), causa placentaria $35.2 \%$, causa inexplicada $7.5 \%$ y causa fetal $5.7 \%$.

Los resultados anatomopatológicos son importantes para identificar causas de óbito fetal tardío. Solo 39 fetos $(36.1 \%)$ registraron tener necropsia. De los fetos con necropsia se pudo determinar que 29 (74\%) de ellos presentaron insuficiencia fetoplacentaria. En tres necropsias no se determinó causa por diferentes problemas (no pasaron la placenta fetos macerados que no se pueden estudiar), (Tabla No 6).

\section{DISCUSIÓN}

La muerte fetal tardía es un problema grave de salud pública con serias repercusiones en la vida familiar. Es de difícil medición debido a la gran diversidad de métodos de clasificación y a las definiciones y análisis adoptados por cada país. En la mayoría de los casos se quedan sin diagnóstico etiológico, y se cata-

\begin{tabular}{|c|c|c|c|}
\hline \multicolumn{4}{|c|}{$\begin{array}{c}\text { TABLA No } 6 \text {. } \\
\text { REPORTE DE NECROPSIA } \\
\text { N=108 }\end{array}$} \\
\hline & & $\mathrm{N}$ & $\%$ \\
\hline \multirow[t]{2}{*}{ Necropsia } & Sí & 39 & 36.1 \\
\hline & No & 69 & 63.9 \\
\hline \multicolumn{4}{|c|}{ Hipoxia fetal extrínseca } \\
\hline \multirow{7}{*}{$\begin{array}{l}\text { Alteraciones de } \\
\text { placenta }\end{array}$} & $\begin{array}{l}\text { Insuficiencia } \\
\text { placentaria }\end{array}$ & 29 & 38.7 \\
\hline & $\begin{array}{l}\text { Infarto } \\
\text { placentario }\end{array}$ & 4 & 5.3 \\
\hline & Necrosis & 1 & 1.3 \\
\hline & $\begin{array}{l}\text { Esclerosis } \\
\text { fibromuscular }\end{array}$ & 1 & 1.3 \\
\hline & Hipoperfusión & 7 & 9.3 \\
\hline & $\begin{array}{l}\text { Depósito } \\
\text { exagerado } \\
\text { de fibrina } \\
\text { perivellosa }\end{array}$ & 17 & 22.7 \\
\hline & $\begin{array}{l}\text { Desprendimiento } \\
\text { de placenta* }\end{array}$ & 4 & 5.3 \\
\hline $\begin{array}{l}\text { Alteraciones del } \\
\text { cordón }\end{array}$ & Prolapso & 1 & 1.3 \\
\hline \multirow[t]{2}{*}{ Anomalías congénitas } & Higroma quístico & 1 & 1.3 \\
\hline & $\begin{array}{l}\text { Malformación } \\
\text { sistema } \\
\text { urinario** }\end{array}$ & 1 & 1.3 \\
\hline $\begin{array}{l}\text { Infecciones } \\
\text { transplacentarias } * * *\end{array}$ & Corioamnionitis & 4 & 5.3 \\
\hline \multicolumn{2}{|l|}{ Hidropsía fetal } & 2 & 2.7 \\
\hline \multicolumn{2}{|l|}{ No determinada $* * * *$} & 3 & 4 \\
\hline
\end{tabular}

*Se incluyeron 2 con hematoma placentario. **Hipoplasia pulmonar bilateral con enfermedad poliquística renal y fascies de Potter.

***No se describe en el reporte tipo de germen causal. ****No se recibe disco placentario en 2 pacientes y signos de maceración en 1 que impidieron el estudio.

logan como muertes inexplicadas. Múltiples factores de riesgo deben tenerse en cuenta en cada población. Es un reto clínico identificar la etiología y los factores de riesgo.

En el presente estudio el grupo etario más frecuente fue de $15-20$ años (34.3\%), este dato difiere de lo encontrado en estudios previos. En una revisión sobre la edad materna y resultados adversos perinatales (29) se reportó la edad avanzada como factor de riesgo independiente. Suarez, et al. en un estudio de muerte fetal tardía realizado en el año 2011 (30) encontraron más frecuencia 
en edades mayores a 20 años. Rivas, en una Clínica de Cartagena en el año 2013 (31), no observó incrementos significativos en el riesgo de muerte fetal en edades extremas de la vida reproductiva, comparados con edades maternas intermedias. Cabe resaltar que su estudio fue realizado para todos los casos de óbito fetal (temprano y tardío).

El estado civil unión libre (86.1\%) y zona de residencia urbana $(58.3 \%)$ son datos similares comparados con estudios previos realizado en la misma ciudad por Rivas et al. (32) y que difieren de revisiones previas donde reportan la zona rural y el estado civil soltera como factores de riesgo, principalmente en países subdesarrollados $(8-10,15,16)$. Con respecto a la raza, en este estudio se encontró con más frecuencia la raza mestiza $(75.9 \%)$, aclarando que en la zona geográfica donde se realizó el estudio la mayoría población es mestiza; escolaridad secundaria completa $(42.6 \%)$, la ocupación ama de casa $(89.8 \%)$, lo cual es diferente a análisis previos. Factores de riesgo como fumar y violencia intrafamiliar no fueron reportados por las pacientes de este estudio.

Otros factores que se relacionan con insuficiencia placentaria como antecedentes de RCIU, pretérminos y óbitos previos no reportaron alta frecuencia en esta investigación. Se encontró un período intergenésico corto y pobre control prenatal como factores de riesgo en las pacientes, además, en la mayoría de ellas el momento de ocurrencia fue el anteparto, lo cual se relaciona con el desconocimiento de los signos de alarma que se enseñan en los controles prenatales.

La edad gestacional en la gran mayoría de las pacientes fue pretérmino (28-36.6 semanas) que concuerda con estudios previos (30), lo cual orienta a que la insuficiencia placentaria puede encontrarse fuertemente asociada a la muerte fetal tardía.

El crecimiento intrauterino es uno de los signos más importantes de bienestar fetal. El IP identifica el recién nacido con poca masa muscular y tejido graso, aun si el peso al nacer es adecuado para la edad gestacional (26). En este estudio se encontró un gran porcentaje por debajo del percentil 10, y, a su vez, 35 pacientes reportaron causas placentarias de muerte fetal.

Con respecto a la concentración de hemoglobina materna, este estudio mostró que las pacientes en su mayoría no presentaban anemia, pero de los casos encontrados con hemoconcentración y anemia severa todos presentaban fetos con percentil de IP menor de 10, lo cual puede relacionarse con RCIU e insuficiencia placentaria. La hemoconcentración se ha asociado con alto riesgo de óbito fetal. Se ha reportado que concentraciones de hemoglobina materna muy alta o muy baja durante el embarazo aumentan el riesgo de nacidos pequeños para la edad gestacional, que es un predictor de muerte fetal.

Sin embargo, la relación entre la concentración de hemoglobina durante el embarazo y el riesgo de muerte fetal no está muy claro. Normalmente en el embarazo se produce expansión del volumen plasmático y disminución de los niveles de hemoglobina, pues de esta manera favorece el crecimiento fetal; si este cambio fisiológico no se da durante el embarazo, se aumenta el riesgo de muerte fetal sin necesidad de que el feto tenga restricción de crecimiento (20). En un estudio poblacional de casos y controles en Suecia se encontró que pacientes con hemoglobinas elevadas detectadas en el principio del control prenatal tenían dos veces más riesgo de presentar óbito fetal (OR 2.0, 95\% C, 1.1-3.8), aun excluyendo pacientes con preeclampsia y eclampsia (20). No obstante, este hallazgo solo pudo explicar el $5.6 \%$ de los óbitos fetales totales.

La obesidad aumenta el riesgo de muerte fetal, principalmente la muerte fetal tardía; el riesgo es independiente de la gravidez y del número de fetos $(22,23)$. Grandes estudios observacionales reportan que las pacientes con sobrepeso y obesidad tienen un $40 \%$ más de riesgo de muerte fetal (22). Las razones para esta asociación son desconocidas, pero se piensa que es debido a los factores conductuales, socioeconómicos y obstétricos. Sin embargo, incluso después de controlar estos factores, la obesidad sigue siendo un factor de riesgo importante para el óbito fetal tardío: el riesgo es independiente de la gravidez y del número de fetos y no impide 
ISSN: 2215-7840, 7(1), enero-junio 2016, Monsalve-Montoya Rosa Edith, Méndez-Rodríguez Rogelio,

Salcedo-Ramos Francisco

que los embarazos múltiples y la obesidad aumenten cuatro veces el riesgo (22). Las características de la población del presente estudio reportaron poca frecuencia de obesidad, pero según los resultados obtenidos el $36 \%$ de las pacientes tienen sobrepeso. Además, al revisar las pacientes con obesidad de este estudio se encontró que 10 (77\%) de ellas presentaban patologías asociadas como trastornos hipertensivos y diabetes.

La mayoría de las pacientes no tenían patologías previas ni relacionadas con el embarazo, pero dentro de las que presentaron patologías, las infecciones genitourinarias y los trastornos hipertensivos son frecuentes. Se revisó en este grupo de pacientes y se encontró que todas reportan insuficiencia fetoplacentaria.

Las causas de óbito fetal tardío más frecuentes fueron las maternas, seguidas por las causas placentarias y dentro de estas, la insuficiencia fetoplacentaria en un alto porcentaje, lo cual coincide con estudios previos $(15,32,33)$. Cuando se analizaron las pacientes con resultados de necropsia se encontró causa e insuficiencia placentaria, que concuerda con el estudio ya citado en la ciudad de Cartagena (32), en donde se evidenció con necropsia causa placentaria en más del $50 \%$ de los casos para óbitos fetales tempranos y tardíos. De las patologías maternas las de mayor frecuencia fueron las infecciones genitourinarias, de esto se deduce que las infecciones ascendentes tienen una gran relación como factor de riesgo en países subdesarrollados.

Las infecciones dan cuenta, aproximadamente, del 10 a $25 \%$ de los óbitos en países desarrollados y de casi la mitad en países subdesarrollados (15). La infección puede llevar a óbito fetal por infección directa a la placenta, por infección severa materna o por infección sistémica directamente al feto. La infección fetal puede producirse por infección ascendente de patógenos del tracto urogenital o por inoculación transplacentaria desde la sangre materna (33). Casi cualquier infección sistémica que ocurre durante el embarazo puede infectar la placenta, pero la muerte fetal como resultado de la infección materna es rara.
El criterio de diagnóstico para determinar si una muerte fetal es debida a infección no está bien definido, y es más complicado por la alta frecuencia de colonización vaginal materna asintomática. Para atribuir la infección como una causa del óbito, se debe evidenciar la infección en la placenta y el feto una vez se hace la necropsia. Aparte de eso, se ha observado que en los óbitos de pacientes con corioamnionitis no se produce respuesta inflamatoria, esto refleja la incapacidad del feto para generar una respuesta inmune en el momento del parto, llevando a la muerte intrauterina (19). Estudios previos han encontrado que mujeres que han tenido un óbito inexplicado tienen un número más alto de "células de T de memoria" (CD45RO) que "células de T nativas" (CD45RA) comparado con las madres de recién nacidos vivos (19). Este hallazgo sugiere que, a pesar de que no haya una evidencia clínica de infección, estas pacientes tuvieron previamente exposición a agentes infecciosos u otros antígenos no identificados que pudieron haber jugado un papel en la muerte fetal.

La malaria es una causa infecciosa común de óbito en áreas endémicas. Parvovirus, cytomegalovirus, toxoplasmosis, listeria y virus de herpes simple son otras causas infecciosas $(33,34)$.

Con respecto a las causas fetales de óbito fetal tardío se reportó un gran porcentaje de fetos sin alteración. Cabe destacar que los estudios de infecciones fetales no son reportados por el laboratorio, pues hasta el momento no son realizados de manera rutinaria, por esta razón las infecciones fetales no se encontraron en ninguno de los fetos estudiados. En la mayoría de los casos no se realizó necropsia, por lo tanto, las malformaciones fetales no se evaluaron en su totalidad y conllevaron a carencia de registros. La baja frecuencia de causa inexplicada para óbito fetal tardío en este estudio difiere de lo descrito en la literatura. Este hecho puede mostrar que hay un gran subregistro de diagnóstico en los países subdesarrollados, situación que puede aumentar la causa inexplicada.

Se identificaron debilidades en este estudio: la clasificación étnica de la población anali- 
zada se realizó con base en las características fenotípicas, teniendo en cuenta que la zona geográfica del estudio limita de forma significativa esta clasificación, pues la población mestiza es mayoría y no se contó en el estudio con análisis genético de la raza. Ninguna de las pacientes declaró ni reportó en la historia clínica ser víctima de maltrato físico o de consumir sustancias tóxicas en el interrogatorio. No se contó con la posibilidad de realizar visita de campo a cada paciente, ni con el acompañamiento de trabajo social para corroborar la información. Las patologías maternas se analizaron de acuerdo al método de recolección de datos descritos. No se contó con exámenes de laboratorio completos para todas las pacientes por igual, lo que hace que enfermedades como las colagenopatías, tiroideos y otras, no se hayan reportado de forma completa. El análisis se realizó de acuerdo a la historia clínica. El laboratorio de patología de la CMRC no cuenta con el material necesario para el análisis de infecciones virales ni de malaria, por lo que no se pudieron determinar de manera más exacta las causas infecciosas.

El estudio aporta datos específicos para la continuidad del análisis de muerte fetal tardía en el país como la importancia de realización de protocolos y guías de manejo de muerte fetal de acuerdo con lineamientos internacionales y recomendaciones de las escuelas de obstetricia, la concientización al personal de salud sobre la búsqueda de los factores de riesgo y el acompañamiento a las gestantes con un adecuado control prenatal.

Las características de la población analizada con óbito fetal tardío en la CMRC de Cartagena de Indias-Colombia mostraron con respecto a factores de riesgo y causas de muerte, algunos datos que difieren de los reportes de literatura para países subdesarrollados. Esto indica que cada institución debe realizar un análisis individual de acuerdo a la población que atiende. Además, la poca adherencia a las normas de salud pública que se evidencia en las pacientes del estudio amerita más atención por parte de las instituciones de salud y entes gubernamentales para reconocer las pacientes en riesgo. La necropsia es un elemento crucial que ayuda a encontrar la causa de muerte en la mayoría de los casos.

\section{CONCLUSIÓN}

Las causas de óbito fetal tardío de las pacientes analizadas en la CMRC de Cartagena de Indias-Colombia en orden de frecuencia son: causa materna, placentaria, inexplicada y fetal.

FINANCIACIÓN: recursos propios de los autores.

CONFLICTO DE INTERESES: ninguno a declarar.

\section{REFERENCIAS BIBLIOGRÁFICAS}

1. World Health Organization. Definitions and indicators in family planning maternal \& child health and reproductive health. World Health Organization, Geneva; 2001.

2. Departamento Administrativo Nacional de Estadística DANE. Glosario de términos para el Sistema de Registro Civil y Estadísticas Vitales. Bogotá DC: DANE, Dirección de censos y demografía; 2013.

3. Lawn JE, Blencowe H, Pattinson R, Cousens S, Kumar R, Ibiebele I, Gardosi J, Day LT, Stanton C. Stillbirths: Where? When? Why? How to make the data count? Lancet. 2011;377(9775):1448-63.

4. Mac Dorman M, Munson M, Kirmeyer S. Fetal and perinatal mortality, United States, 2004. Natl Vital Stat Rep. 2007; 56(3):1-19.

5. Cousens S, Blencowe H, Stanton C, Chou D, Ahmed S, Steinhardt L, Creanga AA, Tunçalp O, Balsara ZP, Gupta S, Say L, Lawn JE. National, regional, and worldwide estimates of stillbirth rates in 2009 with trends since 1995: a systematic analysis. Lancet. 2011;377(1319):1319-30.

6. Instituto Nacional de Salud INS Subdirección de vigilancia y control. Protocolo de vigilancia de mortalidad perinatal primer semestre de 2007. [Internet]; 2007 [cited 2013 junio. Available from: http://www.saludtolima.gov.co/portal/website/publicaciones/direcciones/download. php?id_doc=299.

7. Departamento Administrativo Nacional de Estadística. DANE - Estadísticas Vitales. [Online].; 2013 [cited 2014 noviembre. Available from: http://www.dane.gov.co/index.php/poblacion$y$-registros-vitales/nacimientos-y-defunciones/nacimientos-y-defunciones/118-demograficas/ estadisticas-vitales/4674-defunciones-fetales-2013. 
ISSN: 2215-7840, 7(1), enero-junio 2016, Monsalve-Montoya Rosa Edith, Méndez-Rodríguez Rogelio,

Salcedo-Ramos Francisco

8. Getahun D, Ananth C, Kinzler W. Risk factors for antepartum and intrapartum stillbirth: a population-based study. Am J ObstetGynecol. 2007; 196:499(499):3256-69.

9. Stillbirth Collaborative Research Network Writing Group. Association between stillbirth and risk factors known at pregnancy confirmation. JAMA. 2011; 306(2469):1256-63.

10. Reddy $U$, Laughon $S$, Sun $L$, al. e. Prepregnancy risk factors for antepartum stillbirth in the United States. ObstetGynecol. 2010; 116(1119): 1458-68.

11. Gardosi J, Madurasinghe $V$, Williams $M$, al. e. Maternal and fetal risk factors for stillbirth: population based study. BMJ. 2013; 346(f108).

12. Cunningham F, Leveno K, Bloom S, Hauth J, Rouse D, Spong C. Williams Obstetrics. 23rd ed: The McGraw-Hill Companies; 2010.

13. Fretts R. Etiology and prevention of stillbirth. Am J ObstetGynecol. 2005; 193(1923):5261-72.

14. Reddy U. Prediction and prevention of recurrent stillbirth. ObstetGynecol. 2007; 110(1151):1526-30.

15. Stillbirth Collaborative Research Network Writing Group. Causes of death among stillbirths. JAMA. 2011; 306(2459)1886-96.

16. Ruth Fretts $M$, MPH. Stillbirth epidemiology, risk factors, and opportunities for stillbirth prevention. Clinical Obstetrics and Gynecology. 2010; 53(3):588-96.

17. Pilliod R, Cheng Y, Snowden J, et al. The risk of intrauterine fetal death in the small-forgestational-age fetus. Am J Obstet Gynecol. 2012; 207(318):1-6.

18. Smith R, Maiti K, Aitken R. Unexplained antepartum stillbirth: A consequence of placental aging? Placenta. 2013; 34(4): 310-13.

19. Blackwell $S$, Romero R, Chaiworapongsa $T$, et al. Maternal and fetal inflammatory responses in unexplained fetal death. J Matern Fetal Neonatal Med. 2003; 14(151):45-9. 14 (241).

20. Stephansson O, Dickman PW, Johansson A, Cnattingius S. Maternal hemoglobin concentration during pregnancy and risk of stillbirth. JAMA 2000; 284:2611.

21. Organización Mundial de la Salud. Concentraciones de hemoglobina para diagnosticar la anemia y evaluar su gravedad. Ginebra, Organización Mundial de la Salud, 2011 (WHO/NMH/ NHD/MNM/11.1) (http://www.who.int/vmnis/indicators/haemoglobin_es.pdf, consultado el 13 nov 2014).

22. Chu S, Kim S, Lau J, Schmid C, Dietz P, Callaghan W, et al. Maternal obesity and risk of stillbirth: a metaanalysis. Am J Obstet Gynecol. 2007 Sep; 197(3): p. 223-228.

23. Hamisu M S. Maternal obesity and stillbirth. Seminars in Perinatology. 2011 December; 35(6): 340-4.

24. Atalah E, Castillo C, Castro R. Propuesta de un nuevo estándar de evaluación nutricional en embarazadas. Rev. Med. Chile. 1997; 125: 1429-36.

25. Frøen JF, Gardosi JO, Thurmann A, et al. Restricted fetal growth in sudden intrauterine unexplained death. Acta Obstet Gynecol Scand 2004; 83:801.

26. Milad A, Novoa J, Fabres J, Samamé M, Aspillaga C. Recomendación sobre curvas de crecimiento intrauterino. Rev Chil Pediatr. 2010; 81:264-74.

27. Ovalle S A, Kakarieka W E, Correa P A, Vial P MT, Aspillaga M C. Estudio anatomo-clínico de las causas de muerte fetal. Rev. chil. obstet. ginecol. 2005; 70(5): 303-312.

28. Centro Latinoamericano de Perinatología de la OPS/OMS CLAP. El control prenatal. Uruguay, Montevideo, CLAP (http://www.clap.opsoms.org/web_2005/.../BOLETIN\%2010.pdf, consultado el 13 nov 2014).

29. Carolan M, Frankowska D. Advanced maternal age and adverse perinatal outcome: A review of the evidence. Midwifery. 2011; 27(6):793-801.

30. Suárez-González JA. Caracterización de la mortalidad fetal tardía en Villa Clara. Revista Electrónica de las Ciencias Médicas en Cienfuegos. 2011; 9:12-9.

31. Rivas-Perdomo E. Riesgo de muerte fetal por grupos etarios en una clínica universitaria en Cartagena, Colombia. Rev.cienc.biomed. 2013;4(2):297-301.

32. Rivas-Perdomo E. Óbito fetal: hallazgos de patología en una institución de alta complejidad. Cartagena, Colombia, 2010-2011. Rev Colomb Obstet Ginecol. 2012;63: 376-81.

33. McClure EM. Infection and stillbirth. Seminars in Fetal \& Neonatal Medicine. 2009;14: 182-9.

34. Eleri J. Williams, Nicholas D. Embleton, Julia E. Clark, Mary Bythell, Martin P. Ward Platt, Janet E. Berrington. Viral infections: contributions to late fetal death, stillbirth, and infant death. The Journal of Pediatrics, In Press, Corrected Proof, Available online 16 March 2013.

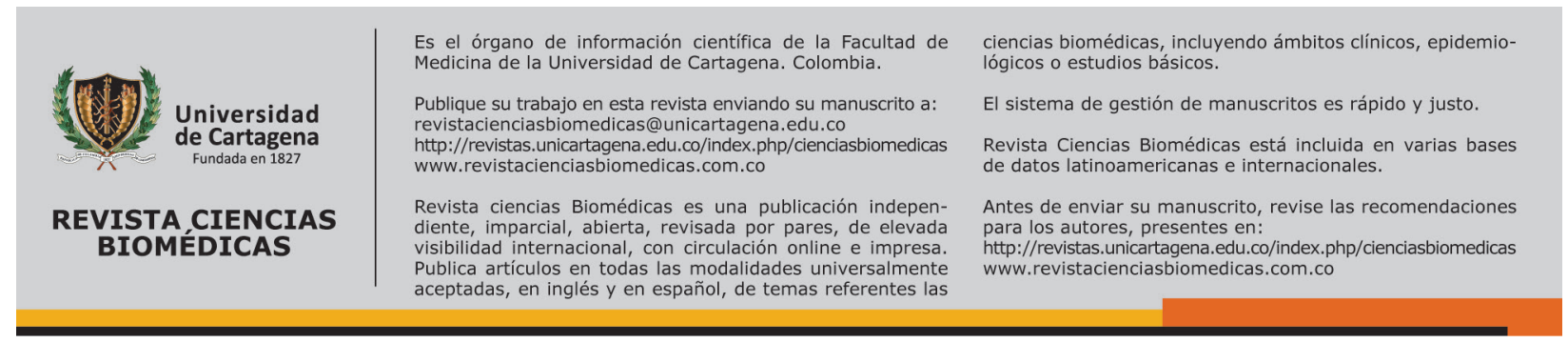

